\title{
Fast and Wide Wavelength-Swept Fiber Optical Parametric Oscillator Based on Dispersion-Tuning
}

\author{
Yue Zhou, Kim K. Y. Cheung, Qin Li, Sigang Yang, P. C. Chui, and Kenneth K. Y. Wong* \\ Photonic Systems Research Laboratory, Department of Electrical and Electronic Engineering, \\ The University of Hong Kong, Pokfulam Road, Hong Kong. \\ *E-mail:kywong@eee.hku.hk
}

\begin{abstract}
We demonstrate a fast and wide tuning wavelength-swept source based on a dispersion-tuned fiber optical parametric oscillator. We achieved the sweep rate of $40 \mathrm{kHz}$ and the wavelength tuning range of $109 \mathrm{~nm}$.

(C)2010 Optical Society of America

OCIS codes: (060.4370) Nonlinear optics, fibers; (190.4970) Parametric oscillators and amplifiers.
\end{abstract}

\section{Introduction}

Wavelength-swept sources have been playing an important role in biomedical imaging [1] and sensing applications [2]. A desirable wavelength-swept source has features such as a wide wavelength range, fast sweep rate, and narrow linewidth in order to capture maximal interferometric information or enhance the spatial resolution. Wavelength-swept sources based on erbium-doped fiber lasers [3] and semiconductor lasers [4] have been investigated comprehensively. However, these techniques can only operate at certain wavelength ranges limited by the material properties of the gain medium. On the other hand, fiber optical parametric oscillator (FOPO) [5] allows swept signal generation in potential regions where practical gain medium may not be available yet. Using the FOPO configuration, a wavelength-swept source has been demonstrated with a $329-\mathrm{nm}$ tuning range, but the cumulative speed is limited to $70,000 \mathrm{~nm} / \mathrm{s}$ [5]. So it is highly desirable to increase the speed of such kind of swept source.

In this paper, we demonstrate a fully fiber-integrated FOPO with a wavelength range of $109 \mathrm{~nm}$, sweep rate of 40 $\mathrm{kHz}$ pumped by a simple clock-modulated pump. Tuning is achieved by changing the clock frequency linearly from 1 $\mathrm{GHz}$ to $1.0006 \mathrm{GHz}$. This scheme has the potential to be a cost-effective source in generating swept signal for biomedical imaging outside the conventional wavelength window.

\section{Experimental Setup}

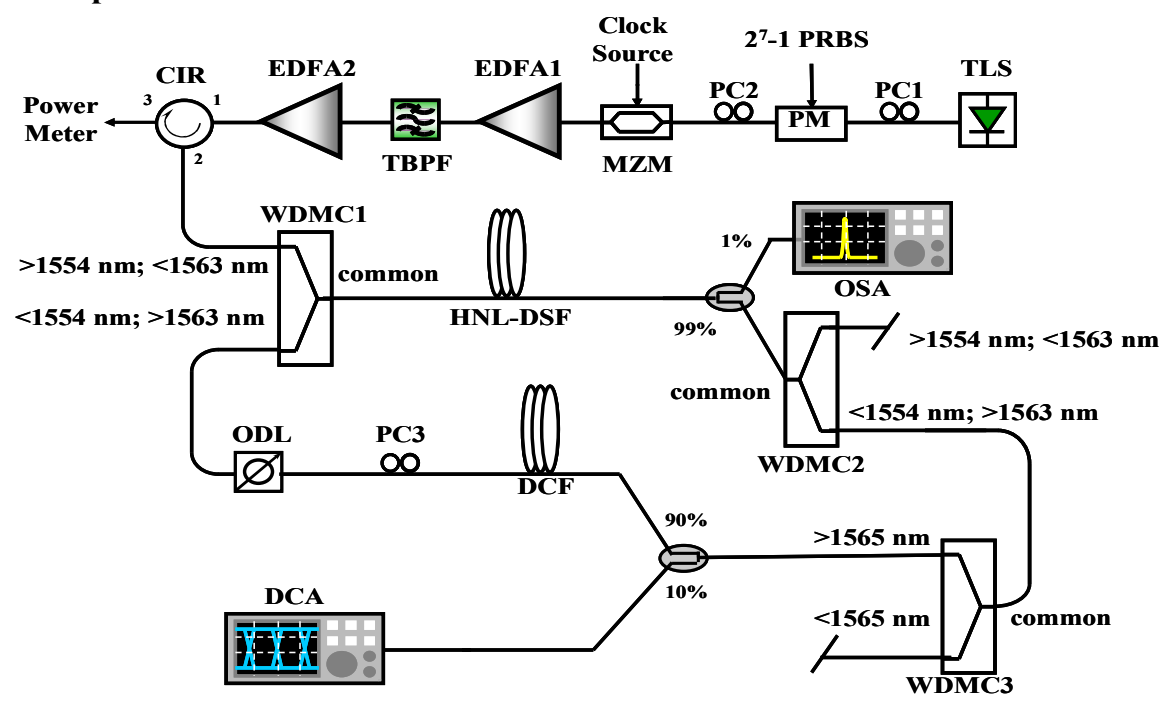

Fig. 1. Experimental setup of the FOPO. TLS: tunable laser source, PC: polarization controller, HNL-DSF: highly-nonlinear dispersion-shifted fiber, OSA: optical spectrum analyzer, MZM: Mach-Zehnder modulator, ODL: optical delay line, CIR: circulator.

The experimental setup of the FOPO is shown in Fig. 1. The pump was generated by a TLS with a fixed wavelength at $1555.5 \mathrm{~nm}$. The output of the laser was phase modulated by a phase modulator (PM) in order to increase the threshold for stimulated Brillouin scattering (SBS). The pump was then intensity modulated by a MZM to produce a sinusoidally modulated pump. Afterwards, the pump was amplified by erbium-doped fiber amplifiers (EDFAs), EDFA1 and EDFA2, and filtered by a tunable bandpass filter (TBPF) with a 1-nm bandwidth to produce a high-power, low-noise pump. The average power of the pump was measured to be $0.85 \mathrm{~W}$ after the EDFA2. The pump then passed 


\section{JTuA30.pdf}

through a circulator, and the reflected power by SBS was monitored using a power meter. It was then coupled into the cavity for parametric amplification through the wavelength-division multiplexing coupler (WDMC1), which had separation wavelengths from $1554 \mathrm{~nm}$ to $1563 \mathrm{~nm}$. Note that the cavity included a 150-m long HNL-DSF as the gain medium which had nonlinear coefficient of $30 \mathrm{~W}^{-1} \mathrm{~km}^{-1}$, zero-dispersion wavelength of $1554 \mathrm{~nm}$ and dispersion slope of $0.02 \mathrm{ps} / \mathrm{nm}^{2} / \mathrm{km}$. The FOPO output spectrum was monitored by an optical spectrum analyzer (OSA) through a 1/99 coupler. WDMC2 was used to filter out the signal and idler by blocking the undesired pump, and WDMC 3 was used to filter away the idler (shorter wavelength sideband) to ensure that only the signal (longer wavelength sideband) can oscillate inside the cavity. A $90 / 10$ coupler in the cavity provided $90 \%$ feedback and $10 \%$ output. Tuning was achieved by adjusting the clock frequency, with no further adjustment of other components. The 200-m dispersion compensation fiber (DCF) in the cavity is used to provide enough dispersion in the cavity thus to perform dispersion-tuning. It had a dispersion of $-96.6 \mathrm{ps} / \mathrm{nm} / \mathrm{km}$ at $1600 \mathrm{~nm}$. The feedback branch passed the PC3 in the cavity, which was used to align the state of polarization (SOP) of the signal with that of the pump, while the optical delay line (ODL) in the cavity was used to adjust the cavity length thus the signal can be synchronized with the pump.

\section{Results and Discussions}
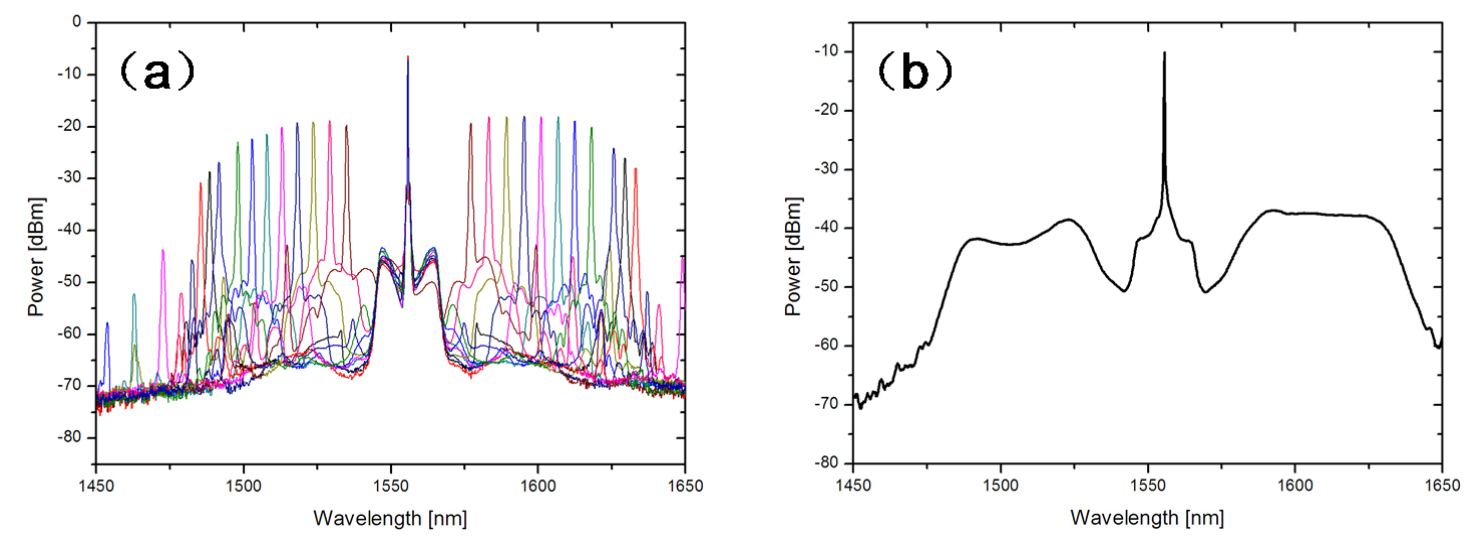

Fig. 2. (a) Static optical spectra measured at HNL-DSF output using OSA; (b) Dynamic optical spectrum measured at HNL-DSF output using OSA. Fig. 2 (a) shows the static optical spectra measured at the HNL-DSF output when the pump is modulated with discrete clock frequency tuned each time. The pump wavelength was fixed at $1555.5 \mathrm{~nm}$ and the pump power remained the same for each tuning. Wavelength tuning is achieved by changing the clock frequency from $1 \mathrm{GHz}$ to $1.0006 \mathrm{GHz}$, while no further adjustment of other components. The achievable tuning range is from $1484 \mathrm{~nm}$ to $1535 \mathrm{~nm}$ and from $1577 \mathrm{~nm}$ to $1635 \mathrm{~nm}$, which is as wide as $109 \mathrm{~nm}$, with a wavelength span of $151 \mathrm{~nm}$. Further tuning is limited by the gain region of the FOPO when the pump wavelength is fixed at $1555.5 \mathrm{~nm}$.

In Fig.2 (b) we show the dynamic optical spectrum when using swept clock frequency scanning linearly from 1 $\mathrm{GHz}$ to $1.0006 \mathrm{GHz}$, with a sweep rate of $40 \mathrm{kHz}$. A flat wide spectrum is achieved. The output has less than $10 \mathrm{~dB}$ power ripples from $1484 \mathrm{~nm}$ to $1534.6 \mathrm{~nm}$, and from $1577.6 \mathrm{~nm}$ to $1634.8 \mathrm{~nm}$, which is as wide as $107.8 \mathrm{~nm}$, matches very well with the 109 -nm tuning range obtained from Fig. 2 (a). The total sweep rate is more than 4,000,000 nm/s, larger than that reported in [5].

\section{Conclusion}

In conclusion, a fast and wide tuning range wavelength swept FOPO is demonstrated. With dispersion-tuning in the cavity, a wide wavelength range of $109 \mathrm{~nm}$ and a fast sweep rate of $40 \mathrm{kHz}$ are obtained. This scheme has the potential to be a cost-effective source in generation swept signal in the non-conventional wavelength bands.

\section{Acknowledgment}

The work described in this paper was partially supported by grants from the Research Grants Council of the Hong Kong Special Administrative Region, China (Project No. HKU 7179/08E and HKU 7183/09E). The authors would also like to acknowledge Sumitomo Electric Industries for providing the HNL-DSF.

\section{References}

[1] S. R. Chinn, E. A. Swanson, and J. G. Fujimoto, Opt. Lett., 22, 340-342 (1997).

[2] Y. Wang, Y. Cui, and B. Yun, IEEE Photon. Technol. Lett., 18, 1539-1541 (2006).

[3] P. F. Wysocki, M. J. F. Digonnet, and B. Y. Kim, Opt. Lett., 15, 879-881 (1990).

[4] S. H. Yun, C. Boudoux, M. C. Pierce, J. F. de Boer, G. J. Tearney, and B. E. Bouma, IEEE Photon. Technol. Lett., 16, 293-295 (2004).

[5] B. P. P. Kuo, N. Alic, P. F. Wysocki, and S. Radic, in Optical Fiber Communication Conference, Postdeadline paper PA9, 2010. 